\title{
Perioperative D-Dimer Levels in Patients with Musculoskeletal Tumors
}

\author{
Takeshi Morii", Kazuo Mochizuki, Masazumi Kotera, Naoaki Imakiire, Takahiro Moriwaki and \\ Kazuhiko Satomi
}

Department of Orthopaedic Surgery, Kyorin University School of Medicine, 6-20-2 Shinkawa Mitaka, Tokyo 181-8611, Japan

\begin{abstract}
Background: D-dimer (DD) levels have been reported as a sensitive but non-specific indicator for deep vein thrombosis (DVT) and pulmonary embolism (PE). Few reports have examined perioperative DD levels in musculoskeletal tumor.
\end{abstract}

\begin{abstract}
Materials/Methods: Subjects comprised 77 patients who had undergone oncological resection of musculoskeletal tumor. DD levels were assessed preoperatively and on postoperative days 1 and 7. Multidetector-row computed tomography (MD-CT) was performed to detect DVT/PE for cases with DD level $>10.0 \mu \mathrm{g} / \mathrm{ml}$.
\end{abstract}

\begin{abstract}
Results: Mean preoperative DD level was $0.84 \mu \mathrm{g} / \mathrm{ml}$. Significant elevation of postoperative DD levels was confirmed. DD levels were significantly changed by various clinical conditions, such as malignancy, age and prosthetic reconstruction. In 4 of 5 cases with postoperative DD levels $>10.0 \mu \mathrm{g} / \mathrm{ml}$, DVT/PE was detected.
\end{abstract}

Conclusion: Activation of the coagulation system by surgery and heterogeneity of DD levels under various clinical conditions in musculoskeletal tumor patients were suggested.

\section{INTRODUCTION}

Activation of coagulation and fibrinolysis is known to be associated with various clinical conditions including malignancy, trauma, surgery and inflammatory disease [1-4]. As such activation results in deep vein thrombosis (DVT) and pulmonary embolism (PE), comprehension of activation status is critical to managing patients with hypercoagulopathy.

D-dimer (DD) is a degradation product of cross-linked fibrin blood clots and reflects fibrin concentration. DD levels are increased in patients with enhanced fibrin formation and have been reported as sensitive indicators for DVT/PE [1, 35]. Orthopaedic surgery itself involves major risks for hypercoagulopathy $[1,6,7]$. In addition, preoperative plasma DD level correlates with tumor stage and prognosis for patients with malignancy [8-12].

Despite the potential clinical significance, few reports have examined DD levels in patients with musculoskeletal tumor. The primary goal of this study was to interpret the basic movement of perioperative DD levels in musculoskeletal tumor patients. In terms of heterogeneity between patients, DD levels might reveal heterogeneity under diverse clinical conditions such as age, tumor origin, location, malignancy or application of prosthesis. The second purpose of this study was thus to analyze the effects of diverse clinical conditions on DD levels in musculoskeletal tumor patients. In addition, clinical data of DVT/PE cases in the present study was disclosed.

*Address correspondence to this author at the Department of Orthopaedic Surgery, Kyorin University, 6-20-2 Shinkawa Mitaka, Tokyo 181-8611, Japan; Tel: +81-422-47-5511; Fax: +81-422-48-4206;

E-mail: t-morii@gb3.so-net.ne.jp or t-morii@kyorin-u.ac.jp

\section{MATERIALS AND METHODS}

Subjects comprised 77 consecutive patients (32 men, 45 women) who underwent oncological resection of musculoskeletal tumor between May 2006 and September 2007. Patients were excluded if the presence of any of the following was identified: pre-existing hypercoagulopathy; recent anticoagulant therapy including prophylaxis of thromboembolic complications during the test period; trauma; inflammatory diseases; and other major surgery. Mean patient age was 45.0 years (range, 11-83 years). Underlying pathology was bone tumor in 32 cases and soft tissue tumor in 45 cases. Tumor location was the lower extremity in 44 cases, the trunk in 22 cases and the upper extremity in 11 cases. For prevention of DVT after surgery, elastic stockings and intermittent pneumatic compression were applied. DD levels were measured using a latex agglutination assay (STA Liatest ${ }^{\mathbb{B}} \mathrm{D}$-Di performed on the STA- $R^{\circledR}$ coagulation analyzer; Roche Diagnostics AG, Rotkreuz, Switzerland). Based on the sensitivity of this assay, DD levels $<0.20 \mu \mathrm{g} / \mathrm{ml}$ were considered as $0.20 \mu \mathrm{g} / \mathrm{ml}$. DD levels were assessed preoperatively and on postoperative days 1 and 7. The impact of diverse clinical conditions including age, tumor origin, site, malignancy and prosthetic reconstruction on DD levels was analyzed. Multidirector-row computed tomography (MD-CT) was indicated for the detection of DVT/PE for cases with DD levels $>10.0 \mu \mathrm{g} / \mathrm{ml}$ in the postoperative period. Clinical profiles of DVT/PE patients were assessed retrospectively. For statistical analysis, Mann-Whitney U-tests, Kruskal Wallis tests, Wilcoxon matched-pair signedrank tests, Spearman rank correlation and $\chi^{2}$ tests were used. Values of $\mathrm{p}<0.05$ were considered statistically significant.

\section{RESULTS}

To analyze pre-existing background bias in samples for this study, correlations between each clinicopathological fac- 
tor (age, tumor origin, site and malignancy) were examined. Patients with malignant tumor were significantly older than patients with benign tumor ( $\mathrm{p}=0.03$, Mann-Whitney U-tests), but no other significant correlations were identified ( $\chi^{2}$ tests).

DD levels at each evaluation time point are summarized in Table 1. Mean preoperative DD level for all subjects was 0.84 $\mu \mathrm{g} / \mathrm{ml}$. Compared to preoperative DD levels, DD levels were significantly elevated on both day 1 ( $p<0.001$, Wilcoxon matched-pair signed-rank test) and day $7(p=0.008)$. Significant correlations were confirmed between preoperative DD and postoperative DD levels on day $1(\mathrm{p}<0.001$, Spearman rank correlation) and day $7(\mathrm{p}<0.001)$.

Significant positive correlations between age and DD levels at each time point were confirmed (preoperative DD, $\mathrm{p}=$ 0.0005 ; postoperative DD on day $1, \mathrm{p}=0.046$; postoperative DD on day $7, p=0.01$, Spearman rank correlation). DD levels at each time point did not differ according to tumor origin (Mann-Whitney U-test). Although DD levels tended to be elevated for trunk or lower extremity cases compared to upper extremity cases, differences were not significant (Kruskal Wallis test). Preoperative DD levels were significantly higher for malignant tumor than for benign tumor $(\mathrm{p}<0.001$, MannWhitney U-test). Postoperative DD levels on day $1(\mathrm{p}<0.001)$ and day $7(\mathrm{p}<0.001)$ were also significantly higher with malignant tumor than with benign tumor. DD levels of patients with prosthetic reconstruction were significantly higher than those without prosthetic reconstruction at each evaluation time point. Due to the limited application of prosthetic reconstruction for malignant bone tumor cases, subclass analysis was performed on the malignant bone tumor group, showing a significant difference in DD level on postoperative day 7 ( $p=0.02$, Mann-Whitney U-test).
DD levels $>10.0 \mu \mathrm{g} / \mathrm{ml}$ were detected in 5 cases postoperatively, and MD-CT was performed for these patients to detect DVT/PE. DVT was detected in 4 of 5 cases. Furthermore, PE was detected in 3 of these 4 cases with DVT. All 4 cases with DVT/PE were silent, and all 4 patients were women. Mean age of the 4 patients with DVT/PE was 65 years (range, 58-79 years). All cases involved malignant tumor (synovial sarcoma in the abdominal muscle, low-grade fibromyxoid sarcoma in the perineal region, undifferentiated high-grade pleomorphic sarcoma in the calf and osteosarcoma in the femur). For all cases, wide resection was performed. For the osteosarcoma case, prosthetic reconstruction was performed. DVT/PE was successfully controlled using heparin and inferior vena cava filter insertion.

\section{DISCUSSION}

To date, measurement of DD has gained a prominent role as a rapid, simple, non-invasive and inexpensive test for ruling out DVT $[1,5,13]$. DD assays are, however, known to show positive results in the presence of common conditions including cancer, surgery, inflammatory disease and trauma $[6,10$, 14-19]. Recent reports have re-confirmed several common properties in the clinical application of DD: variations in baseline levels under different conditions; non-specificity as a marker for DVT/PE; and controversial aspects in the establishment of cut-off values. A recent meta-analysis found that assay sensitivity and negative predictive value were frequently $<90 \%$ and concluded that general use of DD assays as a standalone test for the diagnosis of DVT was not warranted [20]. Another meta-analysis reported that the significance of DD levels in the diagnosis of DVT was the cost-effectiveness when used as a part of algorithms combined with Wells score and ultrasound tests [21].

Table 1. D-dimer Levels in Musculoskeletal Tumor Patients

\begin{tabular}{|c|c|c|c|c|c|c|c|c|c|c|}
\hline \multirow{2}{*}{ Variables } & \multirow{2}{*}{ Number } & \multicolumn{3}{|c|}{$\begin{array}{l}\text { Preoperative D-Dimer } \\
\text { Level }(\mu \mathrm{g} / \mathrm{ml})\end{array}$} & \multicolumn{3}{|c|}{$\begin{array}{c}\text { D-Dimer Level on } \\
\text { Postoperative Day } 1(\mu \mathrm{g} / \mathrm{ml})\end{array}$} & \multicolumn{3}{|c|}{$\begin{array}{c}\text { D-Dimer Level on } \\
\text { Postoperative Day } 7(\mu \mathrm{g} / \mathrm{ml})\end{array}$} \\
\hline & & Mean & Range & $\mathbf{p}$ & Mean & Range & $\mathbf{p}$ & Mean & Range & $\mathbf{p}$ \\
\hline Total cases & 77 & 0.84 & $0.20-4.91$ & & 1.44 & $0.20-9.98$ & & 1.70 & $0.20-17.30$ & \\
\hline \multicolumn{11}{|l|}{ Tumor orgin } \\
\hline Bone & 32 & 1.01 & $0.20-4.91$ & & 1.63 & $0.20-5.92$ & & 1.73 & $0.20-6.95$ & \\
\hline Soft tissue & 45 & 0.73 & $0.20-3.86$ & 0.20 & 1.30 & $0.20-9.98$ & 0.13 & 1.69 & $0.20-17.30$ & 0.28 \\
\hline \multicolumn{11}{|l|}{ Site } \\
\hline Lower extremity & 44 & 0.82 & $0.20-3.31$ & & 1.49 & $0.2-5.92$ & & 1.70 & $0 . .20-8.70$ & \\
\hline Trunk & 22 & 1.04 & $0.20-4.91$ & & 1.70 & $0.32-9.98$ & & 2.28 & $0.20-17.30$ & \\
\hline Upper extremity & 11 & 0.55 & $0.20-2.90$ & 0.13 & 0.72 & $0.20-2.20$ & 0.08 & 0.52 & $0.20-1.30$ & 0.12 \\
\hline \multicolumn{11}{|l|}{ Malignancy } \\
\hline Benign & 30 & 0.34 & $0.20-1.00$ & & 0.71 & $0.20-2.72$ & & 0.48 & $0.20-3.2$ & \\
\hline Malignant & 47 & 1.17 & $0.20-4.91$ & $<0.001$ & 1.90 & $0.25-9.98$ & $<0.001$ & 2.47 & $0.20-17.30$ & $<0.001$ \\
\hline \multicolumn{11}{|l|}{ Prosthesis reconstruction } \\
\hline Yes & 9 & 1.59 & $0.70-3.31$ & & 2.74 & $0.96-5.92$ & & 3.41 & $1.83-6.95$ & \\
\hline No & 68 & 0.75 & $0.20-4.91$ & 0.0005 & 1.27 & $0.20-9.98$ & 0.002 & 1.47 & $0.20-17.30$ & 0.0003 \\
\hline \multicolumn{11}{|c|}{ (In malignant bone tumor cases) } \\
\hline Yes & 9 & 1.59 & $0.70-3.31$ & & 2.74 & $0.96-5.92$ & & 3.41 & $1.83-6.95$ & \\
\hline No & 12 & 1.23 & $0.20-4.91$ & 0.05 & 1.73 & $0.50-3.86$ & 0.14 & 1.62 & $0.20-3.64$ & 0.02 \\
\hline
\end{tabular}


Malignancy [16], chemotherapy [22], reconstruction by prosthesis, reconstruction by plastic surgery, lower extremity predilection, tourniquet application, long-term bed rest and stay in a chronic care facility all represent potential properties of musculoskeletal tumor patients that may lead to abnormalities of coagulation and fibrinolytic activation. Accordingly, patients with musculoskeletal tumor are at increased risk for DVT/PE. To date, however, few studies have examined DD levels in bone and soft tissue tumors [14, 22, 23]. The present investigation represents the first systemic study to describe perioperative DD levels in patients with musculoskeletal tumor. In accordance with previous studies on surgery for other conditions, the present study showed an impact of oncological resection of musculoskeletal tumor on postoperative DD levels.

Heterogeneity in patient characteristics might be marked as a striking limitation in analyzing DD levels in musculoskeletal tumor. Diversity in age [12], resection and reconstruction procedures $[3,24,25]$, tumor location [25] and malignancy [9-12, $18]$ may influence DD levels and incidence of DVT/PE. The present study tried to analyze the impact of several clinicopathological factors on DD levels. As expected, malignancy, age and prosthesis reconstruction proved to have some impact on DD levels, suggesting heterogeneous conditions in DD levels of musculoskeletal tumor patients.

An avoidable limitation of the present study was the retrospective design. The present study used DD levels $<10.0$ $\mu \mathrm{g} / \mathrm{ml}$ as a negative predictor of DVT/PE according to the standard for joint surgery in our institute. Nevertheless, judging from possible differences in condition of the coagulation system between conventional joint disease and musculoskeletal tumor, cut-off values of DD as a predictive value might be reorganized for musculoskeletal tumor patients. Accordingly, prospective regimens regarding detection of DVT/PE using conventional imaging techniques should be considered.

In summary, we retrospectively described perioperative DD levels in 77 consecutive patients with musculoskeletal tumor. Significant activation of the coagulation system under malignant conditions or by surgical procedures in musculoskeletal tumor patients was suggested. Prospective investigation should be considered to determine prevalence rates, cutoff values and time course for DD levels in association with DVT/PE.

\section{REFERENCES}

[1] Kyrle PA, Eichinger S. Deep vein thrombosis. Lancet 2005; 365(9465): 1163-74

[2] Siragusa S, Anastasio R, Giarratano A, et al. Managing cancer patients with acute venous thromboembolism: exploring safe alternatives to hospitalisation. Exp Oncol 2004; 26(3): 192-5.

[3] So AK, Varisco PA, Kemkes-Matthes B, et al. Arthritis is linked to local and systemic activation of coagulation and fibrinolysis pathways. J Thromb Haemost 2003; 1(12): 2510-5.

[4] Wahl WL, Ahrns KS, Zajkowski PJ, et al. Normal D-dimer levels do not exclude thrombotic complications in trauma patients. Surgery 2003; 134(4): 529-32.
[5] Wells PS. Advances in the diagnosis of venous thromboembolism. J Thromb Thrombolysis 2006; 21(1): 31-40.

[6] Maezawa K, Nozawa M, Aritomi K, Kubota M, Shitoto K, Kurosawa H. Changes of D-dimer after total hip arthroplasty in patients with and without intraoperative heparin. Arch Orthop Trauma Surg 2008; 128(1): 37-40.

[7] White RH, Zhou H, Romano PS. Incidence of symptomatic venous thromboembolism after different elective or urgent surgical procedures. Thromb Haemost 2003; 90(3): 446-55.

[8] Altiay G, Ciftci A, Demir M, et al. High plasma D-dimer level is associated with decreased survival in patients with lung cancer. Clin Oncol (R Coll Radiol) 2007; 19(7): 494-8.

[9] Antoniou D, Pavlakou G, Stathopoulos GP, et al. Predictive value of D-dimer plasma levels in response and progressive disease in patients with lung cancer. Lung Cancer 2006; 53(2): 205-10.

[10] Gadducci A, Baicchi U, Marrai R, Ferdeghini M, Bianchi R, Facchini V. Preoperative evaluation of D-dimer and CA 125 levels in differentiating benign from malignant ovarian masses. Gynecol Oncol 1996; 60(2): 197-202.

[11] Kilic M, Yoldas O, Keskek M, et al. Prognostic value of plasma Ddimer levels in patients with colorectal cancer. Colorectal Dis 2008; 10(3): 238-41.

[12] Oya M, Akiyama Y, Okuyama T, Ishikawa H. High preoperative plasma D-dimer level is associated with advanced tumor stage and short survival after curative resection in patients with colorectal cancer. Jpn J Clin Oncol 2001; 31(8): 388-94.

[13] Wells PS. Integrated strategies for the diagnosis of venous thromboembolism. J Thromb Haemost 2007; 5 (Suppl 1): 41-50.

[14] Benevenia J, Bibbo C, Patel DV, Grossman MG, Bahramipour PF, Pappas PJ. Inferior vena cava filters prevent pulmonary emboli in patients with metastatic pathologic fractures of the lower extremity. Clin Orthop Relat Res 2004; 426: 87-91.

[15] Boldt J, Huttner I, Suttner S, Kumle B, Piper SN, Berchthold G. Changes of haemostasis in patients undergoing major abdominal surgery-is there a difference between elderly and younger patients? Br J Anaesth 2001; 87(3): 435-40.

[16] Johnson MJ, Walker ID, Sproule MW, Conkie J. Abnormal coagulation and deep venous thrombosis in patients with advanced cancer. Clin Lab Haematol 1999; 21(1): 51-4.

[17] Mukubo Y, Kawamata M. Higher preoperative D-dimer value remains high postoperatively in patients with rheumatoid arthritis compared with those with osteoarthrosis. J Anesth 2006; 20(1): 51-3.

[18] Satoh T, Oki A, Uno K, et al. High incidence of silent venous thromboembolism before treatment in ovarian cancer. Br J Cancer 2007; 97(8): 1053-7.

[19] Terao M, Ozaki T, Sato T. Diagnosis of deep vein thrombosis after operation for fracture of the proximal femur: comparative study of ultrasonography and venography. J Orthop Sci 2006; 11(2): 146-53.

[20] Heim SW, Schectman JM, Siadaty MS, Philbrick JT. D-dimer testing for deep venous thrombosis: a metaanalysis. Clin Chem 2004; 50(7): 1136-47.

[21] Goodacre S, Sampson F, Stevenson M, et al. Measurement of the clinical and cost-effectiveness of non-invasive diagnostic testing strategies for deep vein thrombosis. Health Technol Assess 2006; 10(15): 1-168.

[22] Osanai T, Tsuchiya T, Ogino T. Deep venous thrombosis during neoadjuvant chemotherapy in a patient with osteosarcoma: a case report. J Orthop Sci 2004; 9(2): 182-5.

[23] Choong PF. Cardiopulmonary complications of intramedullary fixation of long bone metastases. Clin Orthop Relat Res 2003; (415 Suppl): S245-53.

[24] Fujita S, Hirota S, Oda T, Kato Y, Tsukamoto Y, Fuji T. Deep venous thrombosis after total hip or total knee arthroplasty in patients in Japan. Clin Orthop Relat Res 2000; 375: 168-74.

[25] Nicolaides AN, Fareed J, Kakkar AK, et al. Prevention and treatment of venous thromboembolism. International Consensus Statement (guidelines according to scientific evidence). Int Angiol 2006; 25(2): 101-61. 